\title{
Collet-Sicard syndrome as a complication of chronic middle ear infection
}

\author{
Katarina Blažina ${ }^{1}$, Danira Bažadona ${ }^{2}$, Maja Relja ${ }^{3}$ and Danilo Gardijan ${ }^{4}$ \\ ${ }^{1}$ Department of Neurology, University of Zagreb, School of Medicine and University Hospital Centre Zagreb, Kišpatićeva 12, 10000 Zagreb, Croatia \\ ${ }^{2}$ Special Hospital for Chronic Diseases, Josefa Jeruzalema 7, 47250 Duga Resa, Croatia \\ ${ }^{3}$ Department of Neurology, University of Zagreb, School of Medicine and University Hospital Centre Zagreb, Kišpatićeva 12, 10000 Zagreb, Croatia \\ ${ }^{4}$ Department of Radiology, University of Zagreb, School of Medicine and University Hospital Centre Zagreb, Kišpatićeva 12, 10000 Zagreb, Croatia
}

\begin{abstract}
Collet- Sicard syndrome is a rare clinical condition that includes unilateral IX-XII cranial nerves palsy. Various etiological factors may lead to this condition. We present the case of a patient with Collet-Sicard syndrome as a result of chronic middle ear infection and skull base osteomyelitis. Delay in establishing diagnosis led to permanent neurological deficit what emphasize the importance of early recognition.
\end{abstract}

\section{Introduction}

Collet-Sicard syndrome (CSS) is a rare clinical condition comprising $9^{\text {th }}, 10^{\text {th }}, 11^{\text {th }}$ and $12^{\text {th }}$ unilateral cranial nerve palsy, without sympathetic involvement which distinguishes it from Villaret syndrome. When Collet-Sicard syndrome is suspected focal process on the base of the skull must be excluded, first of all primary or metastatic neoplasms but also non-neoplastic disorders like vascular diseases especially dissection or aneurysm of internal carotid artery and sigmoid-jugular complex thrombosis [1]. CSS can be a part of head trauma [2] Lyme disease, idiopathic cranial nerve polyneuropathy and poliartheritis nodosa [1]. It can result from iatrogenic causes like heart surgery and endovascular procedures [3]. Till now only two cases of CSS associated with ear infection have been published $[4,5]$. We present a patient with ColletSicard syndrome due to chronic otitis media and it's propagation to the base of the skull, first presented as cerebrovascular disease.

\section{Case Report}

An 86-year-old man presented to our clinic with sudden unset of dysphonia, dysphagia and consistent pain in the right ear. His medical history included arterial hypertension and diabetes. Eleven and ten years ago he underwent subtotal gastrectomy and left nephrectomy due to gastric and kidney cancer. Neurological examination at the day of the admission revealed dysphonic speech, tongue deviation to the right side, lower palatal arch on the right and weaker sternocleidomastoid muscle on the same side. Fiberendoscopy showed right vocal cord palsy. The rest of the neurological examination was normal, with no autonomic dysfunction. Initial brain multislice computed tomography (MSCT) scan done in emergency department was unremarkable. Subsequently we came across information that two months prior to the admission he underwent right mastoidectomy with polypectomy of the external auditory canal due to chronic inflammation. The pathohistological diagnosis was pyogenic granuloma and due to right ear infection with Pseudomonas aeruginosa he was treated with a second-generation fluoroquinolone, ciprofloxacin during 14 days. Additional MSCT of temporal bones showed mastoid cells filled with mixed absorption coefficient content which was seen in external canal and around ossicles in the middle ear with destruction of the distal mastoid part of the facial nerve bony canal. Posterior wall of the nasopharynx was thickened in the right half, giving an impression of an expansive process destroying outer corticalis of clivus and surrounding internal carotid artery in distal $\mathrm{C} 1$ and proximal C2 segment. On MSCT angiography right internal carotid artery was narrowed in those segments (Figure 1). Bone scintigraphy with technetium-99m-MDP verified pathological

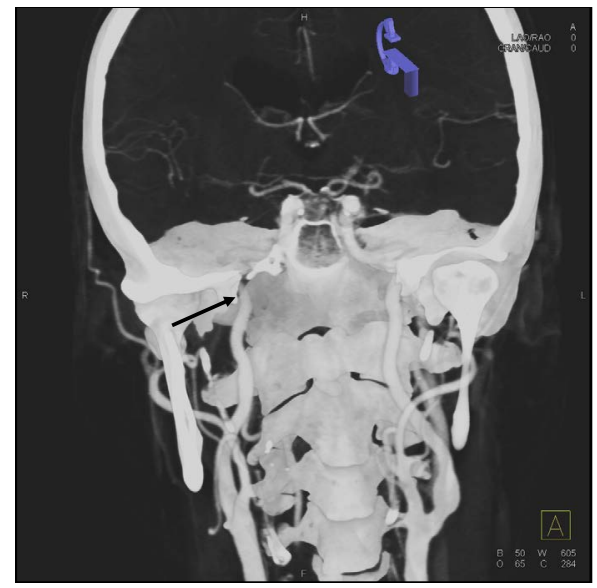

Figure 1a. MSCT angiography reconstruction (coronal plane) shows internal carotid artery narrowing at the entrance of carotid canal (arrow).

Correspondence to: Katarina Blažina M.D., Department of Neurology, University of Zagreb, School of Medicine and University Hospital Centre Zagreb, Kišpatićeva 12, 10000 Zagreb, Croatia, Tel: 00385992491 104; E-mail: katarinabln@gmail.com

Key words: Collet- Sicard syndrome, chronic otitis media, internal carotid artery narrowing, skull base osteomyelitis

Received: July 15, 2016; Accepted: August 01, 2016; Published: August 04, 2016 


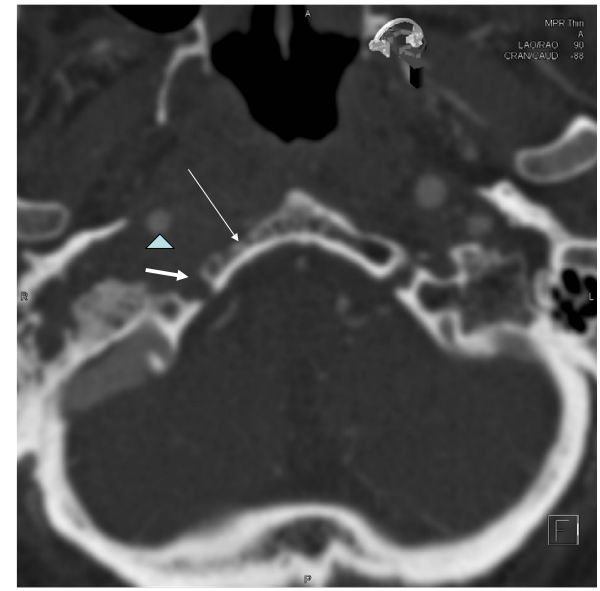

Figure 1b. MSCT reveals significant narrowing of right internal carotid artery (arrowhead), destruction of the hypoglossal canal (thick arrow) and clivus (thin arrow) on the right (axial plane).

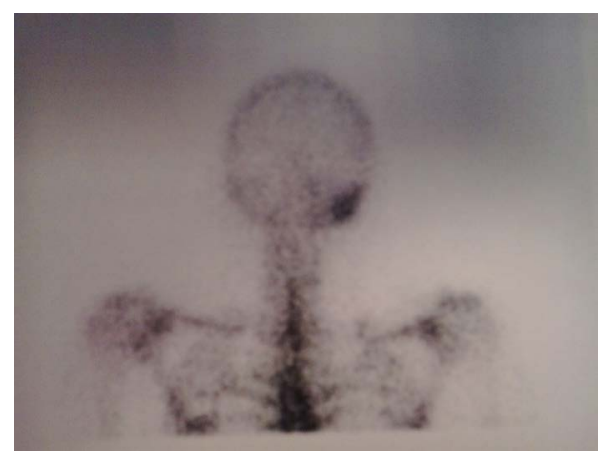

Figure 2. Bone scintigraphy indicate pathological accumulation of the technetium-99mMDP in the right mastoid bone.

accumulation in the lateral right occipital towards mastoid bone and in the $7^{\text {th }}$ thoracic vertebrae (Figure 2). His chest $\mathrm{x}$-ray was unremarkable. Laboratory findings showed microcytic anaemia with low serum iron value ( $8 \mathrm{umol} / \mathrm{L})$ and ferritin $(9.1 \mathrm{ug} / \mathrm{L})$ but high unsatured iron binding capacity ( $67 \mathrm{umol} / \mathrm{L})$. Urea, creatinine, C-reactive protein $(13.1$ $\mathrm{mg} / \mathrm{L}$ ) and fibrinogen values were slightly elevated. Immunoglobulin $\mathrm{G}$ kappa and lambda free light chains were highly elevated (IgG kappa $38.30 \mathrm{mg} / \mathrm{L}$, IgG lambda $59.10 \mathrm{mg} / \mathrm{L}$ ). The rest of the complete blood cell count, liver function enzymes, electrolyte panel, coagulation profile, carcino-embryonic antigen (CEA), alpha-fetoprotein (AFP), carbohydrate antigen 19-9 (CA 19-9), cytokeratin-19 (CYFRA 21-1), neuron-specific enolase (NSE) and prostate-specific antigen (PSA) were within the normal range. He was treated with analgesics: paracetamol (1000 mg per day) and opioid tramadolum (50 mg per day). Because of epipharyngeal process he underwent functional endoscopic surgery with biopsy. Pathohistological analysis revealed mucous parts with mononuclear cell infiltration, but without neoplastic cells. Antibiotic treatment with ciprofloxacin during 21 days has been conducted. Control magnetic resonance imaging of the skull base and temporal bones was done three months after the initial neurological symptoms first occurred. It revealed excessive secretion in the middle ear and destructed mastoid cells. There was intensive post contrast imbibition of the content below dural membrane. It was extended to the cranial edge of the external auditory canal and it represented fibrous change after the surgery. Infiltrative changes of the right pyramidal apex and the body of the sphenoid bone extended to the posterocranial wall of nasopharynx, affecting clivus and circumducting internal carotid artery without compromitation of the blood flow. On one month follow- up examination patient was pain free with persistent residual $9^{\text {th }}-12^{\text {th }}$ cranial nerve palsy on the right side.

\section{Discussion}

CSS is a rare condition that mimics cerebrovascular disease because unilateral cranial nerve involvement. Regarding the prior medical history of arterial hypertension, diabetes and malignancy patient was first considered to have cerebrovascular disease or neoplastic process. He had chronic disease anaemia and overall signs of anergia with normal white blood cell count and just slightly elevated Creactive protein value that first misled us from inflammatory process. Suspicion of malignancy on second MSCT resulted in endoscopic surgery and biopsy. Because all of that appropriate treatment was delayed. Therapeutic approach of CSS is symptomatic depending on underlying etiological cause. Applied antibiotic therapy in our case resolved infection, control imaging showed regression of inflammatory infiltration of the internal carotid artery surroundings. Unfortunately there was no resolution of cranial nerve palsy and neurological deficit was permanent.

Because the condition is rare usually there is a delay in establishing diagnosis. With our report we showed how late diagnosis led to permanent neurological deficit. Early recognition and appropriate symptomatic treatment of undergoing etiological cause may result in significant improvement and functional recovery.

\section{Conflict of Interest}

The authors declare that there is no conflict of interest.

\section{References}

1. Handley TP, Miah MS, Majumdar S, Hussai SS (2010) Collet-Sicard syndrome from thrombosis of the sigmoid-jugular complex: a case report and review of the literature. Int $J$ Otolaryngol. [Crossref]

2. Erol FS, Topsakal C, Kaplan M, Yildirim H, Ozveren MF (2007) Collet-Sicard syndrome associated with occipital condyle fracture and epidural hematoma. Yonsei Med J 48: 120. [Crossref]

3. Prick MJ, Verhagen WI (1992) The Collet-Sicard syndrome as a complication of cardiovascular surgery. J Neurol Neurosurg Psychiatry 55: 741. [Crossref]

4. Climans SA, Melanson M, Desai JA (2013) Case of Collet-Sicard syndrome caused by necrotizing otitis externa. Can J Neurol Sci 40: 268-70. [Crossref]

5. Sibai TA, Ben-Galim PJ, Eicher SA, Reitman CA (2009) Infectious Collet-Sicard syndrome in the differential diagnosis of cerebrovascular accident: a case of head-toneck dissociation with skull-based osteomyelitis. Spine J 9: e6-e10. [Crossref]

Copyright: (C)2016 Blažina K. This is an open-access article distributed under the terms of the Creative Commons Attribution License, which permits unrestricted use, distribution, and reproduction in any medium, provided the original author and source are credited. 